\title{
THE LOOP SPACE PROBLEM AND ITS CONSEQUENCES
}

\author{
BY JAMES P. LIN \\ Communicated March 14, 1975
}

0. Introduction. One of the key results in the study of the topology of Lie groups is the following theorem of Bott [2]:

THEOREM. Let $G$ be a simply connected Lie group. Then $H_{*}(\Omega G ; Z)$ is torsion free.

Bott subsequently coauthored a paper with Samelson [3] which uses this theorem to obtain extensive information about the homotopy and homology of Lie groups. Later, Araki [1] used this result to compute the mod $p$ cohomology of the exceptional groups $E_{7}$ and $E_{8}$ over the Steenrod algebra. Bott's proof depends heavily on the existence of a differential structure on the Lie group.

Shortly after Bott proved this result, it was conjectured that the integral homology of the loops on a finite simply connected $H$-space should be torsion free. We resolve this conjecture for odd primes:

THEOREM 1. Let $X$ be a simply connected finite $H$-space. Then $H_{*}(\Omega X ; Z)$ has no odd torsion.

Actually, we prove this result in a much more general setting. Unlike Bott's proof, which relies heavily on the differential structure, our proof is purely homological and can be applied to $H$-spaces that do not even have the homotopy type of a finite complex.

I wish to thank Bill Browder, John Harper, Richard Kane, J. C. Moore and Alex Zabrodsky for many helpful discussions. I am especially indebted to Richard Kane for pointing out the theorems about the sparseness of the even generators in the $\bmod p$ cohomology ring of an $H$-space.

1. Statement of results. For the remainder of the paper, $X$ will be a two-connected $H$-space having the homotopy type of a CW complex with finitely many cells in each dimension. Furthermore, $p$ will be an odd prime, and we will assume $Q H^{\text {even }}\left(X ; Z_{p}\right)$ is finite dimensional and $\beta_{1} Q H^{\text {even }}\left(X ; Z_{p}\right)=0$.

AMS (MOS) subject classifications (1970). Primary 57F25, 55D45, 55G20; Secondary 57F05, 57F10, $55 \mathrm{~J} 20$. 
Work of Browder [5] shows that any simply connected finite $H$-space satisfies the above conditions. It can be shown that if $X$ is an $H$-space with a finitely generated $\bmod p$ cohomology algebra and the Bockstein spectral sequence collapses after a finite number of steps, then $X$ satisfies the conditions stated above. We have the following theorems:

THEOREM 2. $H_{*}(\Omega X ; Z)$ has no odd torsion.

In the process of proving Theorem 2 we get

Theorem 3. $Q H^{\text {even }}\left(X ; Z_{p}\right)=\Sigma_{l=1}^{\infty} \beta_{1} P^{l} Q H^{2 l+1}\left(X ; Z_{p}\right)$ and $H^{*}(X ; Z)$ has $p$-torsion of order at most $p$.

We now generalize some theorems of Richard Kane [7].

Definition. Let $m$ have $p$-adic expansion

$$
m=\sum_{s \geqslant 0}^{j} m_{s} p^{s}, \quad m_{j} \neq 0,0 \leqslant m_{s}<p .
$$

Then:

$m$ is "unary" if $m_{s}=1$ for every $s \leqslant j$;

$m$ is "binary" if $m_{s}=0$ or 1 for every $s \leqslant j$;

$m$ is "nonbinary" if $m$ is not binary.

Let

$$
\begin{gathered}
v(k)=1+p+p^{2}+\cdots+p^{k}, \quad k \geqslant 1, \\
v(0)=1, \quad v(-1)=0 .
\end{gathered}
$$

Note that if $m$ is binary but not unary, $m$ may be written

$$
m=v(k)+p^{l}+\sum_{s>l}^{j} m_{s} p^{s}, \quad m_{k+1}=0, \quad l>k+1
$$

TheOREM 4. (a) Let $m$ be nonbinary. Then $Q H^{2 m}\left(X ; Z_{p}\right)=0$.

(b) Let $m$ be binary but not unary;

$$
m=v(k)+p^{l}+\sum_{s>l}^{\jmath} m_{s} p^{s}, \quad l>k+1 .
$$

Then

$$
Q H^{2 m}\left(X ; Z_{p}\right)=P^{(m-v(k)) / p} Q H^{2 v(k)+2(m-v(k)) / p}\left(X ; Z_{p}\right) .
$$


Theorem 4 was proven by Kane when $H_{*}\left(X ; Z_{p}\right)$ is associative and $X$ is a simply connected finite $H$-space. We do not need these hypotheses. Theorem 4 may be used to show that the commutator of any two even primitives in $H_{*}\left(X ; Z_{p}\right)$ is zero. Similarly, there are no homology primitive $p$ th powers.

John Harper pointed out this last theorem:

THEOREM 5. The kernel of the Hurewicz map,

$$
\Pi_{n}(X) \otimes Z_{(p)} \stackrel{h_{n} \otimes Z_{(p)}}{\longrightarrow} H_{n}(X ; Z) \otimes Z_{(p)}
$$

is the p-torsion of $\Pi_{n}(X)$.

The proof of these theorems uses techniques developed by Zabrodsky [12] and myself [8], [10]. Details and proofs will appear elsewhere.

\section{REFERENCES}

1. S. Araki, Differential Hopf algebras and the cohomology mod 3 of the compact exceptional groups $E_{7}$ and $E_{8}$, Ann. of Math. (2) 73 (1961), 404-435. MR 35 \#A1372.

2. R. Bott, On torsion in Lie groups, Proc. Nat. Acad. Sci. U.S.A. 40 (1954), 586-588. MR 16, 12 .

3. R. Bott and H. Samelson, On the cohomology ring of $G \mid T$, Proc. Nat. Acad. Sci. U.S.A. 41 (1955), 490-493. MR 17, 182

4. W. Browder, On differential Hopf algebras, Trans. Amer. Math. Soc. 107 (1963), 153-176. MR 26 \#3061.

5. - Torsion in H-spaces, Ann. of Math. (2) 74 (1961), 24-51. MR 23 \#A2201.

6. J. R. Harper, Homotopy groups of H-spaces, Comment. Math. Helv. 47 (1972), $311-331$.

7. R. Kane, On the indecomposables of a finite cohomology Hopf algebra (to appear).

8. J. Lin, H-spaces with finitely generated cohomology algebras, Thesis, Princeton University, 1974.

9. , H-spaces with finitely generated cohomology algebras, Bull. Amer. Math Soc. 80 (1974), 1233-1238.

10. - Torsion in H-spaces. I. (to appear).

11. - H-spaces of exceptional type (to appear).

12. A. Zabrodsky, Secondary operations in the module of indecomposables, Aarhus, 1970, pp. 658-672.

DEPARTMENT OF MATHEMATICS, UNIVERSITY OF CALIFORNIA AT SAN DIEGO, LA JOLLA, CALIFORNIA 92037 